\title{
The Treatment of Diabetes after an Acute Ischemic Stroke
}

\author{
Geert Jan Biessels, MD, PhD ${ }^{1}$ and L Jaap Kappelle, MD, PhD ${ }^{1}$ \\ 1. Professor of Neurology, Department of Neurology, UMC Utrecht Stroke Center and Rudolf Magnus \\ Institute of Neuroscience, University Medical Center Utrecht, Utrecht, The Netherlands
}

\begin{abstract}
People with diabetes are at increased risk of ischemic stroke and outcome after stroke is worse. This paper reviews the treatment of patients with diabetes after ischemic stroke. First, management of hyperglycemia in the acute stage will be addressed. We will make the point that, despite the fact that admission hyperglycemia is a common risk factor for poor outcome after ischemic stroke, there is still much uncertainty about whether intensive glucose-lowering therapy after stroke benefits clinical outcome. Secondary prevention of further cardiovascular events after stroke is the other main topic of this review. We will show that there are important opportunities for the reduction of stroke risk in diabetes, through a rigorous evaluation and treatment of associated risk factors.
\end{abstract}

\section{Keywords}

Diabetes, ischemic stroke, prevention, treatment, outcome

Disclosure: Geert Jan Biessels, MD, PhD, consults for and receives research support from Boehringer Ingelheim. L Jaap Kappelle, MD, PhD, has received speakers honoraria from Bayer and Boehringer Ingelheim.

Acknowledgment: The research of Geert Jan Biessels, MD, PhD, is supported by grant 2010T073 from the Netherlands Heart Foundation.

Received: July 17, 2012 Accepted: August 24, 2012 Citation: US Endocrinology, 2012;8(2):88-92 DOI: 10.17925/USE.2012.08.02.88

Correspondence: Geert Jan Biessels, MD, PhD, Department of Neurology, University Medical Center Utrecht G03.232, PO Box 85500, 3508 GA Utrecht, The Netherlands.

E: g.j.biessels@umcutrecht.nl

Diabetes and ischemic stroke are common conditions that often co-occur. The relationship between diabetes and stroke is bidirectional. on the one hand, people with diabetes have a more than two-fold increased risk of ischemic stroke compared to people without diabetes. ${ }^{1}$ On the other hand, acute stroke can give rise to abnormalities in glucose metabolism, which in turn may affect outcome. ${ }^{2}$ In the current review, which is based on a recent paper from our group in the Lancet Neurology, ${ }^{3}$ we describe the management of diabetes both in the acute stage of stroke and in the longer term, with regard to secondary prevention.

\section{Diabetes and the Risk of Stroke}

A recent meta-analysis of prospective studies including 530,083 participants reported a hazard ratio for ischemic stroke of 2.3 (95\% confidence interval $[\mathrm{Cl}] 2.0-2.7$ ) in people with versus people without diabetes. ${ }^{1}$ Considering that the estimated world-wide prevalence of diabetes in adults is around $10 \%$, this implies that one in eight to nine cases of stroke is attributable to diabetes.

Diabetes is associated with different aetiological subtypes of ischemic stroke, including lacunar and athero- and cardioembolic strokes. ${ }^{4-6}$ Moreover, the risk of atrial fibrillation, the major cause of thromboembolic stroke, is increased by $40 \%$ in diabetes. ${ }^{7}$ Diabetes-associated risk factors for stroke include diabetes-specific factors (e.g. hyperglycemia) and vascular risk factors (e.g. hypertension, dyslipidemia), but also genetic, demographic, and lifestyle factors. The contribution of these factors, many of which are strongly interrelated, is likely to differ according to diabetes type and age.

\section{Hyperglycemia and Stroke Outcome}

Hyperglycemia occurs in 30-40 \% of patients with an acute ischemic stroke. ${ }^{2,8}$ The majority of these patients does not have a known history of diabetes. ${ }^{2}$ In a proportion of patients hyperglycemia reflects pre-existing but unrecognised diabetes, but more often it is due to an acute stress response, commonly named 'stress hyperglycemia'. Stress hyperglycemia is defined as a fasting glucose $>6.9 \mathrm{mmol} / \mathrm{l}$ or a random glucose $>11.1 \mathrm{mmol} / \mathrm{l}$ during hospital stay that spontaneously reverts to normal range after discharge. ${ }^{9}$ Hence, glucose levels at admission generally do not distinguish between stress hyperglycemia and diabetes. In this setting, elevated glycated hemoglobin $\left(\mathrm{HbA}_{1 \mathrm{c}}\right)$ levels $(\geq 6.5 \%)$ can help to identify previously undiagnosed diabetes. ${ }^{9,10}$

Patients with ischemic stroke who are hyperglycemic at admission are at increased risk of poor outcome. Compared to normoglycemic patients, patients with hyperglycemia and a known history of diabetes have an elevated relative risk of in-hospital or 30-day mortality (unadjusted relative risk 2.0 [95\% Cl 0.04-90.1]). ${ }^{2}$ In those without a history of diabetes this risk is $3.3(95 \% \mathrm{Cl} 2.3-4.7) .{ }^{2}$ The association between hyperglycemia and poor outcome primarily involves patients with large-vessel infarction. ${ }^{11}$ In small-vessel 'lacunar' stroke, moderate hyperglycemia has even been associated with improved outcome. ${ }^{8}$ 
There is still uncertainty whether the association between hyperglycemia and poor stroke outcome reflects a causal relation. The acute stress response of the body after stroke is a key factor in the development of post-stroke hyperglycemia and as such it could be argued that hyperglycemia is just an epiphenomenon, indirectly reflecting stroke severity and the condition of the patient. Nevertheless, the link between hyperglycemia and poor outcome appears to be largely independent of other (confounding) factors. ${ }^{2}$ Moreover, experimental and clinical studies have identified several mechanisms through which hyperglycemia could aggravate cerebral damage in ischemic stroke, including impaired recanalisation and reperfusion injury. Figure 1 summarizes these mechanisms. For a more detailed discussion, please see Kruyt et al., 2010.11

\section{Management of Diabetes and Hyperglycemia in Acute Ischemic Stroke}

Plasma glucose levels should be measured on admission in all patients suspected of acute ischemic stroke, regardless of history of diabetes, because glucose levels can direct diagnosis and treatment. There is no reason to exclude patients with a known history of diabetes from receiving thrombolytic therapy. The odds of improvement after thrombolysis are similar in people with or without a history of diabetes (odds ratio [OR] 1.5, $95 \% \mathrm{Cl}$ 1.3-1.6; OR 1.53, $95 \% \mathrm{Cl}$ 1.4-1.6), respectively). ${ }^{12}$ It is less clear if thrombolysis is safe and effective in people with severe hyperglycemia at admission. Trials on intravenous thrombolysis have excluded patients with glucose levels above $22.2 \mathrm{mmol} / \mathrm{l}$. Observational data indicate that hyperglycemia may affect the efficacy of thrombolysis. Transcranial Doppler imaging studies, for example, have demonstrated that hyperglycemia is associated with persistent arterial occlusion after thrombolytic treatment. Others observed that the risk of hemorrhage after thrombolysis is increased for patients with admission glucose levels above $10 \mathrm{mmol} / \mathrm{l}^{13}$

The observed relationship between hyperglycemia and poor outcome in patients with ischemic stroke raises the question whether outcome can be improved by glucose-lowering treatment. Experience in conditions other than stroke, in particular medical or surgical patients at an intensive care unit (ICU), or patients undergoing coronary artery bypass grafting, suggests that glucose-lowering treatment might indeed improve clinical outcome. ${ }^{14-16}$ However, the positive results from these studies have been somewhat tempered by later studies that could not confirm the results. A recent systematic review concluded that there is no consistent evidence that intensive insulin therapy improves health outcomes in hospitalized patients, whereas it is clear that such treatment increases the risk of severe hypoglycemia. ${ }^{17}$

Several studies specifically evaluated the feasibility and safety of glucose-lowering therapy in patients with acute stroke. Although glucose levels can be lowered through various insulin treatment regimens, it can be difficult to achieve stable normoglycemia in the first few days after stroke onset, probably because oral food intake causes fluctuating glucose levels (see Figure 2). ${ }^{18}$ A possible solution to this problem was reported in a study that used continuous tube feeding in combination with intravenous insulin administration. ${ }^{19}$ There also remain important safety issues regarding glucose-lowering treatment, as even with intensive monitoring a substantial number of patients may experience one or more

\section{Figure 1: Hyperglycemia and Infarct Evolution}

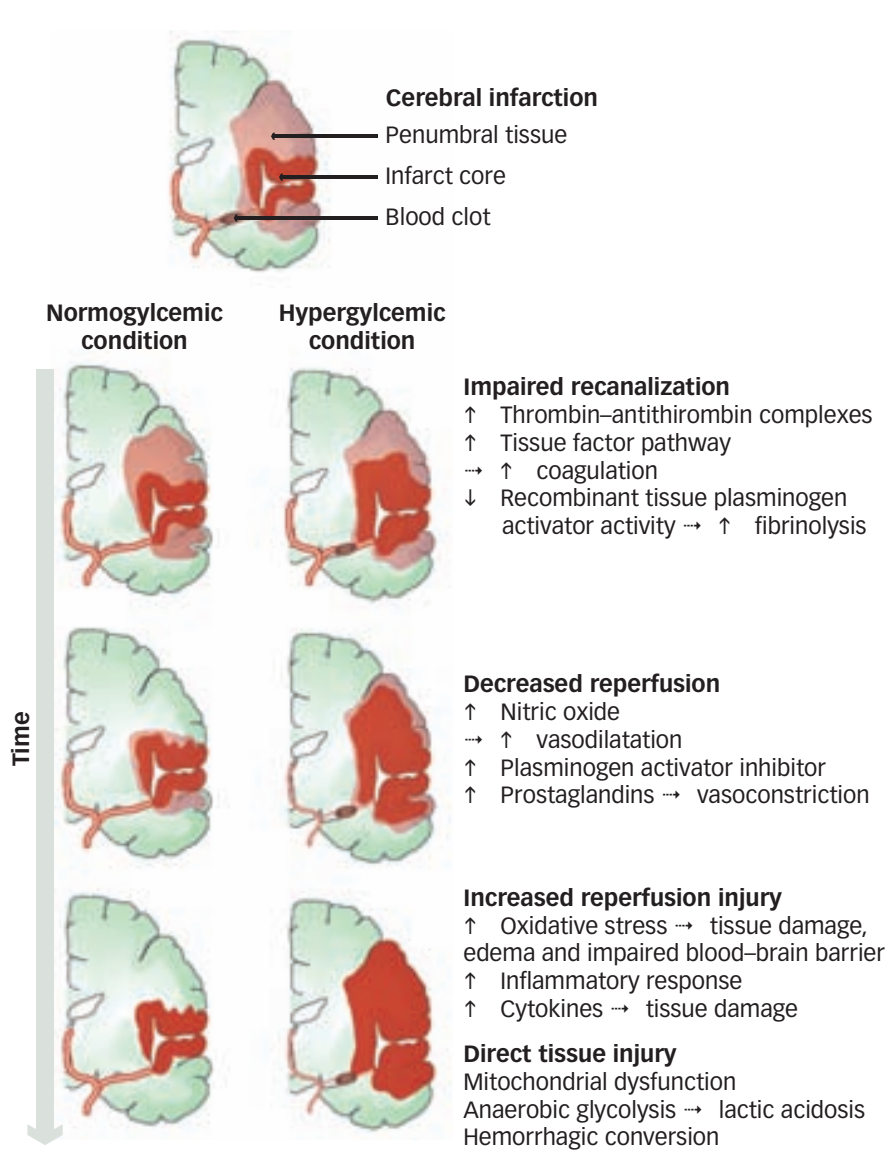

Schematic representation of infarct evolution over time. Hyperglycemia can have deleterious effects on various physiologic processes associated with infarct evolution in patients with acute ischemic stroke. Source: Reproduced with permission from Nature publishing group, Kruyt et al., 2010."1

\section{Figure 2: Glucose-lowering Therapy after Ischemic Stroke}

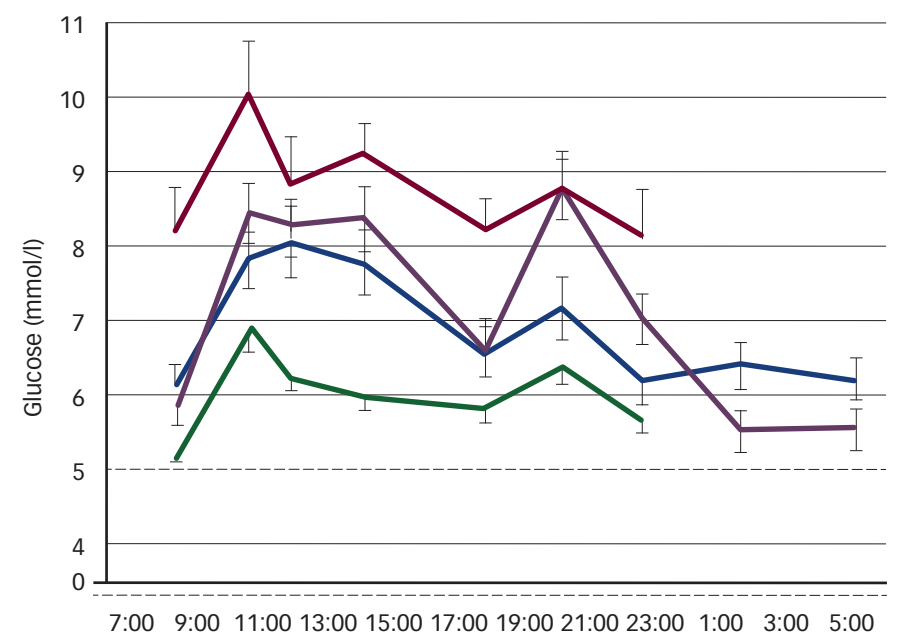

_ Hyperglycemic control group _ Predominantly meal-related insulin group

_ Predominantly basal insulin group _ Normoglycemic control group

Data are from a randomized controlled trial on insulin treatment of post-stroke hyperglycemia. Note the fluctuations in glucose levels over the day in the insulin treated groups.

Source: Reproduced with permission from Vriesendorp et al., 2009. ${ }^{18}$ 


\section{Box 1: Management of Hyperglycemia In Ischemic Stroke}

- Treat hyperglycemia (recommended cut off points: 10.0 or $11.1 \mathrm{mmol} / \mathrm{l}),{ }^{20,21}$ consider that:

- benefit on clinical outcome is not established; and

- Phase II studies show that glucose regulation is feasible, but fluctuations in glucose levels and risk of hypoglycemia are a concern. ${ }^{11,18}$

- Differentiate between stress hyperglycemia and newly diagnosed diabetes. ${ }^{9}$

Source: Based on Luitse et al., 2012. ${ }^{3}$

\section{Box 2: Stroke Prevention In Diabetes}

- Lower blood pressure below 130/80 mmHg. Angiotensin-converting enzyme inhibitors or angiotensin II receptor blockers are recommended as first line treatment. ${ }^{10,35}$

- Prescribe statins. ${ }^{10}$

- Discourage smoking, inactivity, excessive alcohol intake, and obesity.

- Prescribe platelet aggregation inhibitors for secondary prevention in patients with sinus rhythm. ${ }^{38}$

- Apply the $\mathrm{CHA}_{2} \mathrm{DS}_{2}$-VASC score and prescribe warfarin in patients with clinically manifest vascular disease and with atrial fibrillation. ${ }^{10,41}$

- Perform carotid surgery in patients with symptomatic high grade carotid stenosis. ${ }^{46}$

Source: Based on Luitse et al., 2012. ${ }^{3}$

episodes of hypoglycemia. ${ }^{11,18}$ Introduction of continuous glucose monitoring devices and computer-guided treatment algorithms may help to improve the treatment protocols and enhance safety.

Current American Heart Association (AHA) and European Stroke Organisation (ESO) guidelines on ischemic stroke management advise that glucose levels exceeding 11.1 and $10.0 \mathrm{mmol} / \mathrm{l}$, respectively, should trigger insulin therapy (see Box 1). ${ }^{20,21}$ It should be noted, however, that there is as yet no definite evidence that glucose-lowering therapy improves clinical outcome in patients with acute ischemic stroke. Randomized controlled trials specifically targeting patient with stroke have failed to show beneficial effects. A meta-analysis including 1,296 patients with acute stroke from seven trials observed no benefit of intensive insulin therapy over regular care on outcome (OR 1.0, $95 \% \mathrm{Cl}$ 0.8-1.3). The risk of symptomatic hypoglycemia was significantly higher in the intensively treated group insulin (OR 25.9, $95 \%$ Cl 9.2-72.7). ${ }^{22}$ Importantly, the results of this systematic review were mainly determined by the 926 participants of, the Glucose Insulin in Stroke (GIST-UK) trial. ${ }^{23}$ Although this was a landmark trial in this field, it did have some limitations. Patients were treated for only 24 hours, during which mean plasma glucose levels were only $0.57 \mathrm{mmol} / \mathrm{l}$ lower in the intensively treated than the saline treated group. Currently, a new large randomized controlled trial is being initiated that will randomize 1,400 patients to standard care (aiming at glucose levels $<10 \mathrm{mmol} / \mathrm{l}$ ) or intravenous insulin treatment (aiming at glucose levels between 4.4 to $7.2 \mathrm{mmol} / \mathrm{l}$ ) for 72 hours after stroke (Johnston et al., SHINE, NCT01369069). The study will use an insulin infusion protocol that has been proven safe and feasible in a pilot study. ${ }^{24}$

\section{Secondary Prevention}

Several risk factors for stroke in patients with diabetes are potentially modifiable, in particular life style factors, glucose levels, blood pressure and dyslipidemia (see Box 2). These risk factors have been targeted in several large randomized controlled trials. Neurologists often distinguish between primary prevention (e.g. prevention of first stroke) and secondary prevention (e.g. prevention after transient ischemic attack (TIA) or ischemic stroke). Although this review is written form the perspective of secondary prevention, it should be acknowledged that the literature on prevention of cardiovascular events in people with diabetes does not always allow to make this distinction.

Lifestyle probably has the largest impact on the risk of stroke. Therefore, smoking, obesity, inactivity, excessive alcohol intake, and unhealthy diets should be strongly discouraged in patients with diabetes, even more so after the occurrence of a TIA or stroke. Lifestyle modification in patients with diabetes is associated with a substantial decline in stroke incidence (hazard ratio [HR] 0.62, $95 \% \mathrm{Cl} 0.39-0.98$ ). ${ }^{25}$ Moreover, modest weight loss (5-10\% of body weight) in patients with type 2 diabetes has been associated with a significant improvement of cardiovascular risk factors and glycemic control. ${ }^{26,27}$

\section{Glucose-lowering Therapy}

Three large long-term trials have compared the effects of intensive versus standard glycemic control on cardiovascular outcomes in relatively high-risk participants with longstanding type 2 diabetes. Two of these trials showed no difference with intensive glycemic control. ${ }^{28,29}$ The Action to Control Cardiovascular Risk in Diabetes Study (ACCORD) study terminated its glycemic control study after 3.7 years due to an increased mortality in the intensive treatment group (target $\mathrm{HbA}_{1 \mathrm{c}}<42 \mathrm{mmol} / \mathrm{mol}$, $6 \%$ ). ${ }^{30}$ Participants assigned to the intensive therapy group were subsequently switched to the standard control group (target $\mathrm{HbA}_{1 \mathrm{c}}$ between 53 and $64 \mathrm{mmol} / \mathrm{mol}, 7-7.9 \%$ ) and followed for on average 1.2 years. Both before and after the transition the risk for non-fatal stroke was similar. Reasons for the higher mortality in the intensive therapy group remained unclear. ${ }^{31}$ In a meta-analysis including 34,533 patients with type 2 diabetes no beneficial effects of tight glycemic control versus standard glycemic control could be demonstrated on stroke rates during a mean treatment period of five years ( $\mathrm{HR} 0.96,95 \% \mathrm{Cl} 0.8-1.1){ }^{32}$

The Diabetes Control and Complications Trial (DCCT) showed that 1,422 patients with type 1 diabetes, who were treated with intensive control of glucose levels during six and a half years, had a reduced risk of cardiovascular events over a mean follow up of 17 years of $57 \%$ (95\% $\mathrm{Cl} 12-79 \%)$ as compared to patients receiving conventional treatment. ${ }^{33}$ However, strokes were rare, with only one event in the intensive treatment group and five in the conventional treatment group. ${ }^{33}$

Taken together, the results of available studies do not provide sufficient evidence that stroke prevention will be improved by intensive glucose-lowering therapy either in type 1 or in type 2 diabetes. Clinicians 
should balance the risk of (recurrent) hypoglycemia against the advantages of a lower $\mathrm{HbA}_{1 c}$ level, taking into account patient's age, the duration of diabetes and patient's comorbidities.

\section{Vascular Risk Factors}

In patients with type 2 diabetes lowering of blood pressure has a large impact on the risk of future stroke. A recent meta-analysis of patients with type 2 diabetes or impaired glucose tolerance evaluated the results of 37,736 patients from 13 trials that aimed to compare control of blood pressure $\leq 135 \mathrm{mmHg}$ with $\leq 140 \mathrm{mmHg} .{ }^{34}$ More intensive control of blood pressure was associated with a $10 \%$ reduction in all-cause mortality (OR 0.90, $95 \% \mathrm{Cl} 0.83-0.98)$ and a $17 \%$ reduction in strokes (OR 0.83, $95 \% \mathrm{Cl} 0.73-0.95$ ) as compared with standard treatment. This difference was mainly driven by the trials that aimed at a systolic pressure between 130 and $135 \mathrm{mmHg}$. Control of blood pressure below $130 \mathrm{mmHg}$ was associated with a greater reduction in stroke, but there was a $40 \%$ increase in serious adverse events with no benefit for cardiac, renal, and retinal outcomes. ${ }^{34}$ Most guidelines recommend a blood pressure below 130/80 mmHg for patients with diabetes..$^{10}$ The choice of antihypertensive drugs is probably less important than the target levels. Currently, angiotensin-converting enzyme inhibitors or angiotensin $\|$ receptor blockers are often recommended as the first line medication. ${ }^{10,35}$

The Heart Protection Study demonstrated in a post hoc analysis that a daily dose of $40 \mathrm{mg}$ simvastatin administered to 5,963 patients with type 2 diabetes, of whom half did not have any evidence of arterial occlusive disease, was associated with a $28 \%(95 \% \mathrm{Cl} 8-44 \%)$ reduction in ischemic stroke, independent of the baseline lipid levels. ${ }^{36}$ In 9,795 patients with type 2 diabetes, of whom 7,664 patients had no history of cardiovascular disease, micronised fenofibrate $200 \mathrm{mg}$ once daily as compared with placebo reduced the risk of cardiovascular events (HR 0.89, $95 \% \mathrm{Cl}$ 0.80-0.99), including ischemic stroke (HR 0.91, $95 \%$ $\mathrm{Cl}$ 0.73-1.1). ${ }^{37}$ Based on this evidence statins are recommended for secondary prevention in all patients with type 2 diabetes and in most patients also for primary prevention, depending on their 10-year cardiovascular risk. ${ }^{10}$ There is no consensus about the choice of statins.

\section{Antithrombotic Treatment}

No major trial has studied the effectiveness of antithrombotic medication for the secondary prevention of stroke specifically in a diabetic population. A meta-analysis that investigated the efficacy of antithrombotic agents in more than 5,000 patients with diabetes concluded that these drugs reduced both coronary events and ischemic stroke to a similar degree as in patients without diabetes. ${ }^{38}$ Hence, current policy for prescription of antithrombotic drugs for secondary prevention is similar in patients with or without diabetes. A meta-analysis on the use of aspirin for primary prevention in patients with diabetes demonstrated no benefits with respect to the reduction of serious vascular events, including stroke. ${ }^{39}$ Although the impact of antithrombotic treatment on the prevention of future cardiovascular events is relatively low as compared to rigorous control of risk factors, it should be considered in each patient with diabetes at risk for future vascular complications.

Diabetes is a risk factor for atrial fibrillation..$^{40}$ In addition, diabetes increases the risk of embolic complications in patients with atrial fibrillation as is reflected in the $\mathrm{CHA}_{2} \mathrm{DS}_{2}$-VASC score. ${ }^{41}$ Therefore, patients with diabetes and atrial fibrillation should receive platelet aggregation inhibitors if they have none of the other risk factors included in the $\mathrm{CHA}_{2} \mathrm{DS}_{2}-\mathrm{VASC}^{41}$ and anticoagulants in all other cases. ${ }^{42}$ Recently, dabigatran, rivaroxaban, and apixaban have been proven to protect patients with atrial fibrillation as good, or even better than warfarin, but the definite role of these new antithrombotic agents in patients with a recent TIA or minor ischemic stroke remains to be established. ${ }^{43}$

\section{Carotid Surgery}

Carotid endarterectomy for secondary stroke prevention in patients with high-grade stenosis of the carotid artery is effective, but has not been specifically investigated in diabetic patients. Both the peri-procedural and long-term risks are higher in patients with diabetes than in non-diabetic patients, ${ }^{44,45}$ but this should not be a reason to withhold the surgery in this patient group.

\section{Conclusion}

Diabetes is a key factor in the risk of ischemic stroke and in stroke outcome. Evaluation of diabetes status and blood glucose levels is therefore an essential component of the diagnostic work-up after stroke. Optimal glucose management in the acute stage of stroke is still an area of uncertainty that warrants further investigations. Rigorous management of vascular risk factors can prevent strokes in people with diabetes
1. Sarwar N, Gao P, Seshasai SR, et al., Diabetes mellitus, fasting blood glucose concentration, and risk of vascular disease: a collaborative meta-analysis of 102 prospective studies, Lancet 2010;375(9733):2215-22.

2. Capes SE, Hunt D, Malmberg K, et al., Stress hyperglycemia and prognosis of stroke in nondiabetic and diabetic patients: and prognosis of stroke in nondlabetic and diabetic pa

3. Luitse MJ, Biessels GJ, Rutten GE, Kappelle LJ, Diabetes, hyperglycaemia, and acute ischaemic stroke, Lancet Neurol, 2012;11(3):261-71.

4. Jackson C, Sudlow C, Are lacunar strokes really different? A systematic review of differences in risk factor profiles between lacunar and nonlacunar infarcts, stroke, 2005;36(4):891-901.

5. Cui R, Iso H, Yamagishi $\mathrm{K}$, et al., Diabetes mellitus and risk of stroke and its subtypes among Japanese: the Japan public health center study, Stroke, 2011;42(9):2611-4.

6. Ohira T, Shahar E, Chambless LE, et al., Risk factors for ischemic stroke subtypes: the Atherosclerosis Risk in Communities study, Stroke, 2006:37(10):2493-8.

7. Huxley RR, Filion KB, Konety S, Alonso A, Meta-analysis of cohort and case-control studies of type 2 diabetes mellitus and risk of atrial fibrillation, Am I Cardiol, 2011:108(1):56-62.

8. Uyttenboogaart M, Koch MW, Stewart RE, et al., Moderate hyperglycaemia is associated with favourable outcome in acute lacunar stroke, Brain, 2007;130(Pt 6):1626-30.

9. Dungan KM, Braithwaite SS, Preiser JC, Stress hyperglycaemia Lancet, 2009:373(9677):1798-807.

10. American Diabetes Association, Standards of medical care in diabetes--2011, Diabetes Care, 2011;34(Suppl. 1):S11-S61.

11. Kruyt ND, Biessels GJ, Devries JH, Roos YB, Hyperglycemia in acute ischemic stroke: pathophysiology and clinical management, Nat Rev Neurol, 2010;6(3):145-55.

12. Mishra NK, Ahmed N, Davalos A, et al., Thrombolysis outcomes in acute ischemic stroke patients with prior stroke and diabetes mellitus, Neurology, 2011;22;77(21):1866-72.

13. Ahmed N, Davalos A, Eriksson N, et al., Association of admission blood glucose and outcome in patients treated with intravenous thrombolysis: results from the Safe Implementation of Treatments in Stroke International Stroke Thrombolysis Register (SITS-ISTR), Arch Neurol, 2010;67(9):1123-30.

14. Van den Berghe $\mathrm{G}$, Wouters $\mathrm{P}$, Weekers $\mathrm{F}$, et al., Intensive insulin therapy in the critically ill patients, N Eng/ I Med, 2001;345(19):1359-67.
15. Van den Berghe G, Wilmer A, Milants I, et al., Intensive insulin therapy in mixed medical/surgical intensive care units: benefit versus harm, Diabetes, 2006;55(11):3151-9.

16. Furnary $\mathrm{AP}, \mathrm{Gao} \mathrm{G}$, Grunkemeier $\mathrm{GL}$, et al., Continuous insulin infusion reduces mortality in patients with diabetes undergoing coronary artery bypass grafting $J$ Thorac Cardiovasc Surg, 2003;125(5):1007-21.

17. Kansagara $D, F u$, Freeman $M$, et al., Intensive insulin therapy in hospitalized patients: a systematic review, Ann Intern Med, 2011;154(4):268-82.

18. Vriesendorp TM, Roos YB, Kruyt ND, et al., Efficacy and safety of two 5 day insulin dosing regimens to achieve strict glycaemic control in patients with acute ischaemic stroke I Neurol Neurosurg Psychiatry, 2009;80(9):1040-3.

19. Kruyt ND, Biessels GJ, Vriesendorp TM, et al., subjecting acute ischemic stroke patients to continuous tube feeding and an intensive computerized protocol establishes tight glycemic control, Neurocrit Care, 2010;12(1):62-8.

20. Guidelines for management of ischaemic stroke and transient ischaemic attack 2008, Cerebrovasc Dis, 2008;25(5):457-507.

21. Adams HP Jr, del Zoppo G, Alberts MJ, et al., Guidelines for the early management of adults with ischemic stroke: a 
guideline from the American Heart Association/American Stroke Association Stroke Council, Clinical Cardiology Council, Cardiovascular Radiology and Intervention Council, and the Atherosclerotic Peripheral Vascular Disease and Quality of Care Outcomes in Research Interdisciplinary Working Groups: The American Academy of Neurology affirms the value of this guideline as an educational tool for neurologists, circulation 2007;115(20):e478-e534

22. Bellolio MF, Gilmore RM, Stead LG, Insulin for glycaemic control in acute ischaemic stroke, Cochrane Database syst Rev 2011;(9):CD005346

23. Gray CS, Hildreth AJ, Sandercock PA, et al., Glucosepotassium-insulin infusions in the management of post-stroke hyperglycaemia: the UK Glucose Insulin in Stroke Trial (GIST-UK), Lancet Neurol, 2007;6(5):397-406.

24. Johnston KC, Hall CE, Kissela BM, et al., Glucose Regulation in Acute Stroke Patients (GRASP) trial: a randomized pilot trial, Stroke, 2009;40(12):3804-9.

25. Sone $\mathrm{H}$, Tanaka $\mathrm{S}$, limuro $\mathrm{S}$, et al., Long-term lifestyle intervention lowers the incidence of stroke in Japanese patients with type 2 diabetes: a nationwide multicentre randomised controlled trial (the Japan Diabetes Complications Study), Diabetologia, 2010;53(3):419-28.

26. Look AHEAD Research Group, Wing RR, Long-term effects of a lifestyle intervention on weight and cardiovascular risk factors in individuals with type 2 diabetes mellitus: four-year results of the LOOK AHEAD trial, Arch Intern Med, 2010;170(17):1566-75.

27. Wing RR, Lang W, Wadden TA, et al., Benefits of modest weight loss in improving cardiovascular risk factors in overweight and obese individuals with type 2 diabetes, Diabetes Care, 2011;34(7):1481-6.

28. ADVANCE Collaborative Group, Patel A, MacMahon S, et al., Intensive blood glucose control and vascular outcomes in patients with type 2 diabetes, $N$ Eng / I Med 2008;12;358(24):2560-72.
29. Duckworth W, Abraira C, Moritz T, et al., Glucose control and vascular complications in veterans with type 2 diabetes, N Eng/ J Med, 2009;360(2):129-39.

30. Gerstein HC, Miller ME, Byington RP, et al., Effects of intensive glucose lowering in type 2 diabetes, N Engl I Med, 2008;358(24):2545-59.

31. ACCORD Study Group, Gerstein $\mathrm{HC}$, Miller ME, Genuth $\mathrm{S}$, et al., Long-term effects of intensive glucose lowering on cardiovascular outcomes, N Eng/ J Med, 2011;364(9):818-28

32. Boussageon $\mathrm{R}$, Bejan-Angoulvant $\mathrm{T}$, Saadatian-Elahi $\mathrm{M}$ et al., Effect of intensive glucose lowering treatment on all cause mortality, cardiovascular death, and microvascular events in type 2 diabetes: meta-analysis of randomised controlled trials, BMJ, 2011;343:d4169.

33. Nathan DM, Cleary PA, Backlund JY, et al., Intensive diabetes treatment and cardiovascular disease in patients with type 1 diabetes, N Engl J Med, 2005;353(25):2643-53.

34. Bangalore S, Kumar S, Lobach I, Messerli FH, Blood pressure targets in subjects with type 2 diabetes mellitus/impaired fasting glucose: observations from traditional and bayesian random-effects meta-analyses of randomized trials, circulation, 2011;123(24):2799-810, 9.

35. Goldstein LB, Bushnell CD, Adams RJ, et al., Guidelines for the primary prevention of stroke: a guideline for healthcare professionals from the American Heart Association/American Stroke Association, Stroke, 2011:42(2):517-84.

36. Collins R, Armitage J, Parish $\mathrm{S}$, et al., MRC/BHF Heart Protection Study of cholesterol-lowering with simvastatin in 5963 people with diabetes: a randomised placebo-controlled trial, Lancet, 2003;361(9374):2005-16.

37. Keech A, Simes RJ, Barter P, et al., Effects of long-term fenofibrate therapy on cardiovascular events in 9795 people with type 2 diabetes mellitus (the FIELD study): randomised controlled trial, Lancet, 2005;366(9500):1849-61.
38. Antithrombotic Trialists' Collaboration, Collaborative meta-analysis of randomised trials of antiplatelet therapy for prevention of death, myocardial infarction, and stroke in high risk patients, BMJ, 2002;324(7329):71-86

39. De Berardis G, Sacco M, Strippoli GF, et al., Aspirin for primary prevention of cardiovascular events in people with diabetes: meta-analysis of randomised controlled trials, BM! 2009;339:b4531.

40. Huxley RR, Alonso A, Lopez FL, et al., Type 2 diabetes, glucose homeostasis and incident atrial fibrillation: the Atherosclerosis Risk in Communities study, Heart, 2012;98(2):133-8.

41. Lip GY, Nieuwlaat R, Pisters R, et al., Refining clinical risk stratification for predicting stroke and thromboembolism in atrial fibrillation using a novel risk factor-based approach: the euro heart survey on atrial fibrillation, chest, 2010;137(2):263-72

42. European Heart Rhythm Association European Association for Cardio-Thoracic Surgery, Camm AJ, Kirchhof P, Lip GY, et al., Guidelines for the management of atrial fibrillation: the Task Force for the Management of Atrial Fibrillation of the European Society of Cardiology (ESC), Europace, 2010:12(10):1360-420

43. Rothwell PM, Algra A, Amarenco P, Medical treatment in acute and long-term secondary prevention after transient ischaemic attack and ischaemic stroke, Lancet, 2011;377(9778):1681-92.

44. Cunningham EJ, Bond R, Mehta Z, et al., Long-term durability of carotid endarterectomy for symptomatic stenosis and risk factors for late postoperative stroke, Stroke, 2002;33(11):2658-63.

45. Debing E, Aerden D, Van den Brande P, Diabetes mellitus is a predictor for early adverse outcome after carotid endarterectomy. Vasc Endovascular Surg, 2011;45(1):28-32.

46. Rerkasem K, Rothwell PM, Carotid endarterectomy for symptomatic carotid stenosis, Cochrane Database Syst Rev 2011;(4):CD001081. 